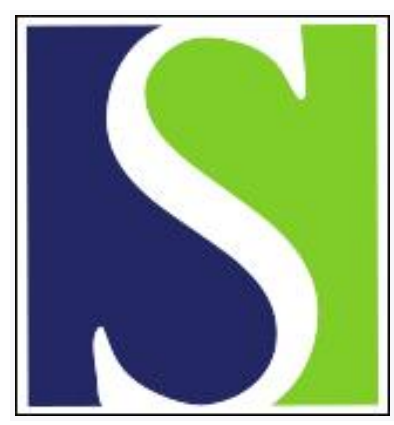

Scand J Work Environ Health 2002;28(1):49-57

https://doi.org/10.5271/sjweh.646

Issue date: Feb 2002

Exploration of asthma risk by occupation - extended analysis of an incidence study of the Finnish population

by Karjalainen A, Kurppa K, Martikainen R, Karjalainen J, Klaukka T

Affiliation: Finnish Institute of Occupational Health, Topeliuksenkatu 41 a A, FI-00250 Helsinki, Finland. antti.karjalainen@occuphealth.fi

Refers to the following texts of the Journal: 1996;22(6):451-456 1998;24(4):262-269

The following articles refer to this text: 2009;35(1):64-73;

2022;48(1):1-3

Key terms: asthma; asthma risk; epidemiology; Finland; incidence study; occupation; occupational asthma

This article in PubMed: www.ncbi.nlm.nih.gov/pubmed/11871852 


\title{
Exploration of asthma risk by occupation - extended analysis of an incidence study of the Finnish population
}

\author{
by Antti Karjalainen, MD, ${ }^{1}$ Kari Kurppa, MD, ${ }^{1}$ Rami Martikainen, MSc, ${ }^{1}$ Jussi Karjalainen, MD, ${ }^{2}$ Timo \\ Klaukka, MD ${ }^{3}$
}

\begin{abstract}
Karjalainen A, Kurppa K, Martikainen R, Karjalainen J, Klaukka T. Exploration of asthma risk by occupation extended analysis of an incidence study of the Finnish population. Scand J Work Environ Health 2002;28(1):49 57.
\end{abstract}

Objectives The objective of the study was to determine asthma risks at the most-detailed level of occupational classification in a previously described nationwide follow-up study that included the entire employed workforce of Finland.

Methods In Finland, persons with clinically verified persistent asthma are registered for medication reimbursement within the national health insurance scheme. Data were combined from three national registers, and all 25to 59-year-old employed Finns were followed for asthma incidence in 1986-1998. Altogether 49575 cases were detected. A log-linear model was used to estimate the relative risks of asthma for 275 nonadministrative occupations in comparison with administrative work (33 occupations).

Results A significantly increased risk was found for either men or women in 125 occupations. For the men, the risk was highest among bakers, laundry workers, shoemakers and repairers, tanners, fell mongers and pelt dressers, and metal plating and coating workers. For the women, the risk was highest among shoemakers and repairers, railway and station personnel, jewelry engravers, engineroom crew, molders, round-timber workers, and bakers.

Conclusions The results suggest that the work-related excess of asthma incidence is much more widely spread across the labor force than has been previously thought. A great number of occupations deserves to be targeted for in-depth studies focusing on the determinants of asthma excess and on possibilities for better asthma control among asthmatics working in these occupations. The large work-relatedness of asthma incidence should also raise public health interest because of the economic losses incurred and the potential for prevention.

Key terms epidemiology, incidence, occupational asthma, risk.

Although more than 200 specific agents at worksites are known to cause asthma (1), knowledge on etiologies and risks for occupational asthma still remains incomplete, and the population burden is poorly known even for the recognized causative agents. One reason for such a limited understanding is that asthma epidemiology is hampered by peculiar methodological difficulties that distinguish it from the epidemiology of many other chronic diseases. (See reference 2.) Difficulties for epidemiologic studies are created by problems of definition, conclusive case ascertainment, and the variable clinical course of asthma.

Large population-based comparisons of asthma incidence between occupational groups could provide deeper insight into the extent of work-related asthma. The variation of disease incidence among different occupational groups may suggest hypotheses on risks that have thus far remained unrecognized. Specific in-depth epidemiologic studies could then be focused on such occupations. Conventional strategies for such explorations are, however, ineffective, the annual incidence of adultonset asthma being below $0.3 \%$.

In Finland linkages of national registers allow for an efficient exploration of the differences in asthma incidence between occupational groups. Persons with clinically well-established persistent asthma are eligible for medication reimbursement within the national health insurance scheme and are therefore registered. We have

1 Finnish Institute of Occupational Health, Department of Epidemiology and Biostatistics, Helsinki, Finland.

2 Tampere University Hospital, Department of Respiratory Medicine, Tampere, Finland.

3 Social Insurance Institution, Helsinki, Finland.

Reprint requests to: Antti Karjalainen, Finnish Institute of Occupational Health, Topeliuksenkatu 41 a A, FIN-00250 Helsinki, Finland. [E-mail: antti.karjalainen@occuphealth.fi] 
conducted a nationwide population follow-up in order to calculate occupation-specific incidence rates and relative risks of adult-onset asthma. On the basis of this follow-up, we recently reported estimates of the attributable fraction for work-related factors in persistent adult-onset asthma (3). The attributable fraction of work was $29 \%$ for men and $17 \%$ for women. This excess was mainly due to an increased incidence rate in the major occupational categories of agricultural, manufacturing, and service work. The nearly 50000 incident cases of asthma of the study make exceptionally detailed occupation-specific analyses possible. The aim of our present paper was to extend the previously reported coarse analysis to the most-detailed level of occupational classification in order to estimate the occupation-specific relative risks of asthma and to analyze the importance of certified occupational asthma in the occupation-specific excess.

\section{Subjects and methods}

\section{Study population and design}

The study consisted of a follow-up of the employed Finnish population during 1986-1998. Technically it consisted of three cohorts from consecutive national population censuses. All employed Finns without preexisting asthma and between the ages of 25 and 59 years on 31 December 1985, 1990, and 1995 were classified according to their occupation and followed for asthma during 1986-1990, 1991-1995, and 1996-1998, respectively. There were $0.99,1.01$, and 0.88 million employed men and $0.90,0.95$, and 0.83 million employed women under follow-up in the 1985, 1990 and 1995 cohorts, respectively.

The follow-up was done using the unique personal identification code and two national registers (the Medication Reimbursement Register of the Social Insurance Institution of Finland and the Finnish Register of Occupational Diseases). Duplicate notifications between the registers were identified using the personal identification code. The matching of the registers to the population census according to the personal identification code failed in only $0.01 \%$ of the cases. For each follow-up period the cases and person-years were calculated according to the occupation the person held at the start of the follow-up. The study protocol was approved by the ethics committees of the participating institutions.

\section{Definition and ascertainment of cases}

In Finland, asthma is one of the chronic diseases that a person can receive compensation for medication at a higher than ordinary level. To receive the compensation the patient has to apply for the entitlement from the Social Insurance Institution and provide a medical certificate written by a chest physician. (For children the physician must be a pediatrician.) For the application to be granted, the disease has to fulfill the diagnostic and severity criteria of asthma, including objective data of reversible bronchial obstruction and a typical and persistent pattern of disease (3). The national health insurance, which includes a drug reimbursement system, covers the entire population and consequently almost all Finnish patients with asthma fulfilling the criteria are identifiable in the register. The only exception concerns patients with recognized occupational asthma who receive full compensation for medication from Statutory Accident Insurance. The latter group of patients is centrally registered by the Finnish Register of Occupational Diseases. The recognition of occupational asthma necessitates a diagnosis of asthma made by a chest physician and individual evidence of a causal association between a specific workplace exposure and the disease.

A person was defined as an asthmatic case if he or she had received the right to be reimbursed for asthma medication from the Social Insurance Institution (95\% of the cases) or had been notified for recognized occupational asthma to the Finnish Register of Occupational Diseases (5\% of the cases). The date of the application (Social Insurance Institution) or the date of the notification (Finnish Register of Occupational Diseases) was used as the date of diagnosis.

Among those with special reimbursement rights for asthma medication, the reliability of the asthma diagnosis is high. In a random sample of working-aged asthmatics with reimbursement rights, clinically established asthma was present for $99 \%$ (3).

\section{Classification of occupation}

Information on employment status and current occupation is recorded for every person in the Finnish population census. The occupation was known for $99 \%$ of the employed in each of the three censuses. The occupational code is an extended classification of the International Standard Classification of Occupations published by the International Labour Office in 1958. In the analyses we used the following 10 major occupational groups and their 307 occupational categories: technical, physical science, social science, humanistic and artistic work (73 occupations), administrative, managerial and clerical work (33 occupations), sales work (12 occupations), agriculture, forestry, commercial fishing (16 occupations), mining and quarrying work (4 occupations), transport and communications work (24 occupations), manufacturing and related work (114 occupations), service work (30 occupations), military work (1 occupation), and unknown occupation. 


\section{Statistical analysis}

The tasks performed and the exposures in a given occupation may differ between men and women. There is also a gender difference in the overall incidence of adultonset asthma (4). Therefore all the analyses were performed separately for both genders. The incidence rate for each major occupational group and each occupation was calculated by summing up the cases and personyears from the three census cohorts and dividing the total number of cases by the accumulated person-years under follow-up. Incidence rate ratios (hereafter referred to as relative risks) and their $95 \%$ confidence intervals were estimated for each of the 275 nonadministrative occupations by a log-linear model adjusted for age at the start of the follow-up (25-39, 40-49, 50-59 years). Those employed in administrative, managerial, and clerical work (hereafter referred to as administrative work) were used as a reference group with little exposure to dusts, fumes, vapors, or other workplace air pollution.

\section{Control of confounding}

Adjustment for the follow-up period did not influence the occupation-specific relative risks, and this variable was not included in the model. Separate analyses were performed using as reference only the lower-level administrative employees (secretaries, office clerks, bookkeepers, and similar occupations). The incidence rate of asthma in this reference group was similar to that of administrative workers in general, and consequently the relative risks for the exposed occupations did not change. Hence only the results based on the reference group of all administrative work are presented.

\section{Results}

There were 20777 incident cases of asthma among the men and 28798 among the women during the followup, of which 1224 cases among the men and 1240 cases among the women were cases of recognized occupational asthma.

The relative risk (RR) was estimated for all 275 of the nonadministrative occupations. For 125 of them, a significantly increased risk was found for either the men or the women (table 1). In 45 of them, the risk was significantly increased for both genders, in 35 the risk was significantly increased for one gender and nonsignificantly increased for the other, and 32 represented occupations in which the workforce consisted mainly of one gender only (ie, less than 5 cases in the other gender). In 12 of the occupations listed in table 1, the risk was significantly increased for one gender but nonsignificantly decreased for the other. For only one of the occupations was the risk significantly increased for one gender and significantly decreased for the other (table $1)$.

Table 1. Risk occupations for adult-onset asthma among employed Finns (25-59 years of age) in 1986-1998. In addition to all major occupational groups, the table includes all occupations with a significantly increased relative risk (RR) and $\geq 5$ cases for either gender. ( $\mathrm{RR}=$ relative risk, $95 \% \mathrm{Cl}=95 \% \mathrm{Cl}$ confidence interval)

\begin{tabular}{|c|c|c|c|c|c|c|c|c|}
\hline \multirow[t]{2}{*}{ Occupation a } & \multicolumn{4}{|c|}{ Men } & \multicolumn{4}{|c|}{ Women } \\
\hline & N & $\begin{array}{c}\text { Popula- } \\
\text { tion b }\end{array}$ & $\mathrm{RR}^{\mathrm{c}}$ & $95 \% \mathrm{Cl}$ & N & $\begin{array}{c}\text { Popula- } \\
\text { tion b }\end{array}$ & $\mathrm{RR}^{\mathrm{c}}$ & $95 \% \mathrm{Cl}$ \\
\hline Technical, physical science, social science, humanistic and artistic work (0) & 3369 & 214450 & 1.05 & $0.98-1.12$ & 7024 & 262063 & 1.06 & $1.03-1.10$ \\
\hline Civil engineering technicians $(010)$ & 349 & 16610 & 1.28 & 1.14-1.45 & 77 & 2533 & 1.28 & $1.02-1.60$ \\
\hline Technicians in chemical engineering (014) & 132 & 6400 & 1.24 & $1.04-1.49$ & 24 & 637 & 1.51 & $1.01-2.25$ \\
\hline Technicians in mining and metallurgy (015) & 43 & 1534 & 1.65 & $1.22-2.23$ & 2 & 33 & 2.41 & $0.60-9.64$ \\
\hline Draftsmen and survey assistants (018) & 43 & 2081 & 1.62 & $1.20-2.20$ & 63 & 2542 & 0.98 & $0.77-1.26$ \\
\hline Laboratory assistants (028) & 47 & 2729 & 1.19 & $0.89-1.59$ & 231 & 7682 & 1.20 & 1.05-1.37 \\
\hline Psychiatric nurses (035) & 43 & 1757 & 1.78 & $1.31-2.41$ & 81 & 3006 & 1.01 & $0.81-1.25$ \\
\hline Assistant nurses and attendants (036) & 24 & 1208 & 1.72 & $1.15-2.57$ & 784 & 24525 & 1.27 & $1.18-1.37$ \\
\hline Other occupations related to medical and nursing work (039) & 42 & 1433 & 1.84 & $1.35-2.50$ & 16 & 675 & 0.90 & $0.55-1.48$ \\
\hline Health inspectors (042) & 6 & 619 & 0.59 & $0.26-1.30$ & 16 & 340 & 1.87 & 1.14-3.05 \\
\hline Directors and nursing staff at child day care centers (055) & 4 & 436 & 0.88 & $0.33-2.35$ & 236 & 9089 & 1.16 & $1.02-1.32$ \\
\hline Other occupations in education and training (059) & 33 & 1314 & 1.56 & $1.10-2.21$ & 53 & 2022 & 1.16 & $1.02-1.32$ \\
\hline Lay preachers (061) & 11 & 272 & 2.41 & $1.33-4.36$ & 6 & 139 & 1.43 & $0.64-3.17$ \\
\hline Religious social work (062) & 14 & 746 & 1.34 & $0.79-2.27$ & 77 & 2230 & 1.34 & $1.07-1.68$ \\
\hline Performing artists and vocalists (086) & 21 & 596 & 2.43 & $1.58-3.74$ & 16 & 572 & 1.13 & $0.69-1.85$ \\
\hline Social workers, child day care center staff, etc (091) & 81 & 4226 & 1.34 & $1.07-1.67$ & 1255 & 38474 & 1.27 & $1.19-1.35$ \\
\hline Administrative, managerial and clerical work (1) & 1275 & 79087 & 1.00 & (reference) & 5235 & 202751 & 1.00( & reference) \\
\hline Sales work (2) & 1106 & 66541 & 1.14 & $1.05-1.23$ & 2429 & 79116 & 1.13 & $1.08-1.18$ \\
\hline Retailers and leaseholders (201) & 209 & 10462 & 1.22 & $1.05-1.41$ & 359 & 9657 & 1.32 & $1.19-1.47$ \\
\hline Commercial travelers and salesmen (220) & 236 & 13579 & 1.17 & $1.02-1.35$ & 62 & 2808 & 0.89 & $0.69-1.14$ \\
\hline Shop personnel (231) & 343 & 21448 & 1.15 & $1.02-1.30$ & 1663 & 52014 & 1.15 & $1.09-1.21$ \\
\hline Service station attendants (234) & 46 & 2325 & 1.37 & $1.02-1.83$ & 36 & 1397 & 1.00 & $0.72-1.74$ \\
\hline
\end{tabular}


Table 1. Continued.

\begin{tabular}{|c|c|c|c|c|c|c|c|c|}
\hline \multirow[t]{2}{*}{ Occupation a } & \multicolumn{4}{|c|}{ Men } & \multicolumn{4}{|c|}{ Women } \\
\hline & N & $\begin{array}{l}\text { Popula- } \\
\text { tion b }\end{array}$ & $\mathrm{RR}^{\mathrm{c}}$ & $95 \% \mathrm{Cl}$ & N & $\begin{array}{l}\text { Popula- } \\
\text { tion b }\end{array}$ & $\mathrm{RR}^{\mathrm{c}}$ & $95 \% \mathrm{Cl}$ \\
\hline Agriculture, forestry, commercial fishing (3) & 3670 & 97052 & 2.12 & $1.99-2.26$ & 3279 & 58617 & 1.84 & $1.76-1.92$ \\
\hline Farmers, silviculturists, horticulturists $(300)$ & 2774 & 64180 & 2.32 & $2.17-2.48$ & 1912 & 34070 & 1.90 & $1.80-2.00$ \\
\hline Farm supervisors (302) & 12 & 354 & 1.98 & $1.12-3.49$ & - & 27 & & . \\
\hline Forestry supervisors (303) & 91 & 3613 & 1.39 & $1.12-1.72$ & - & 43 & . & . \\
\hline Livestock breeders (305) & 4 & 306 & 1.06 & $0.40-2.82$ & 23 & 630 & 1.94 & $1.29-2.93$ \\
\hline Fur farmers (306) & 56 & 1435 & 2.35 & $1.80-3.08$ & 16 & 291 & 1.83 & $1.12-3.00$ \\
\hline Reindeer breeders (307) & 17 & 560 & 1.87 & $1.16-3.02$ & - & 21 & & \\
\hline Agricultural workers $(310)$ & 273 & 7323 & 2.31 & $2.03-2.64$ & 1227 & 20230 & 1.89 & $1.77-2.01$ \\
\hline Gardeners and park workers (311) & 43 & 1775 & 1.62 & $1.19-2.19$ & 57 & 2017 & 1.03 & $0.79-1.33$ \\
\hline Other occupations in agriculture, horticulture and animal husbandry (319) & 3 & 89 & 2.44 & $0.79-7.58$ & 11 & 170 & 2.54 & $1.41-4.59$ \\
\hline Forestry workers and log floaters (340) & 346 & 14287 & 1.54 & $1.37-1.74$ & 7 & 192 & 1.20 & $0.57-2.52$ \\
\hline Mining and quarrying work, etc (4) & 93 & 3028 & 1.95 & $1.58-2.40$ & 2 & 69 & 1.01 & $0.25-4.02$ \\
\hline Miners, shot firers, etc (400) & 38 & 1049 & 2.16 & $1.56-2.98$ & - & 6 & 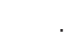 & . \\
\hline Concentration plant wo & 12 & 329 & 2.36 & $1.34-4.17$ & - & 6 & & . \\
\hline Other miners and quarrymen (490) & 29 & 1045 & 1.83 & $1.27-2.65$ & 1 & 30 & 1.21 & $0.17-8.58$ \\
\hline Transport and communications work (5) & 1938 & 94398 & 1.31 & $1.22-1.40$ & 796 & 23331 & 1.22 & $1.13-1.31$ \\
\hline Engineroom crew (511) & 11 & 477 & 1.51 & $0.83-2.73$ & 7 & 108 & 2.58 & $1.23-5.41$ \\
\hline tram drivers (540) & 1321 & 61700 & 1.35 & $1.25-1.46$ & 86 & 2380 & 1.33 & $1.07-1.65$ \\
\hline Railway and station personnel (traffic department) (550) & 117 & 5069 & 1.43 & $1.19-1.73$ & 13 & 137 & 3.25 & $1.89-5.60$ \\
\hline Road transport supervisors (563) & 59 & 2426 & 1.49 & $1.15-1.94$ & 5 & 562 & 0.37 & $0.16-0.90$ \\
\hline Telephone switchboard operators (572) & 6 & 263 & 1.72 & $0.77-3.84$ & 142 & 4117 & 1.24 & $1.05-1.46$ \\
\hline Newspaper delivery work (581) & 16 & 931 & 1.21 & $0.74-1.99$ & 100 & 2190 & 1.57 & $1.29-1.92$ \\
\hline Office receptionists (concessionaires) and messengers (582) & 56 & 2416 & 1.48 & $1.13-1.93$ & 52 & 1162 & 1.63 & $1.24-2.14$ \\
\hline Manufacturing and related work (6/7) & 7836 & 334935 & 1.56 & $1.47-1.65$ & 3270 & 88555 & 1.33 & $1.27-1.39$ \\
\hline Weaving machine operators (602) & 7 & 261 & 1.84 & $0.87-3.86$ & 54 & 1244 & 1.50 & 97 \\
\hline Textile & 1 & 12 & 5.99 & $0.84-$ & 18 & 355 & 1.62 & \\
\hline tresses $(610)$ & 7 & 161 & 1.83 & $0.87-3.85$ & 64 & 1762 & 1.31 & $1.02-1.67$ \\
\hline Industrial se & 5 & 123 & 2.63 & $1.09-6.33$ & 394 & 13044 & 1.10 & $0.99-1.22$ \\
\hline Other cutting, sewing and upholstering occupations (619) & 6 & 153 & 2.73 & $1.23-6.09$ & 36 & 1269 & 1.02 & $0.73-1.42$ \\
\hline Shoemakers and shoe repairers (620) & 12 & 284 & 2.79 & $1.58-4.93$ & 6 & 50 & 4.32 & $1.94-9.61$ \\
\hline Shoe sewers, leather cutters for footwear and other footwear workers (621) & 1) 20 & 694 & 1.87 & $1.20-2.91$ & 48 & 2210 & 0.77 & $0.58-1.02$ \\
\hline Metal smelting furnacemen (630) & 51 & 2040 & 1.62 & $1.22-2.14$ & 3 & 61 & 6.31 & 2.03-19.6 \\
\hline Smiths (633) & 19 & 430 & 2.37 & $1.51-3.74$ & 1 & 26 & 1.22 & $0.17-8.69$ \\
\hline Molders (634) & 26 & 1271 & 1.33 & $0.90-1.97$ & 12 & 166 & 2.56 & $1.45-4.51$ \\
\hline Wire and pipe drawers & 10 & 305 & 2.11 & $1.13-3.94$ & 2 & 42 & 1.59 & $0.40-6.35$ \\
\hline Other occupations in smelting, metallurgical and foundry work (639) & 44 & 1580 & 1.84 & $1.36-2.49$ & 8 & 301 & 0.91 & $0.45-1.82$ \\
\hline Jewelry engravers (645) & 3 & 104 & 1.73 & $0.56-5.38$ & 6 & 79 & 2.77 & $1.25-6.18$ \\
\hline Turners, toolmakers and machine-tool setters $(650)$ & 328 & 14800 & 1.52 & $1.35-1.72$ & 30 & 842 & 1.25 & $0.87-1.79$ \\
\hline Fitter-assemblers, etc $(651)$ & 352 & 17413 & 1.41 & $1.25-1.59$ & 7 & 321 & 0.84 & $0.40-1.76$ \\
\hline Machine and engine mechanics (652) & 389 & 20584 & 1.30 & $1.16-1.45$ & 7 & 283 & 0.94 & $0.45-1.97$ \\
\hline Sheet metal workers $(653)$ & 385 & 14615 & 1.86 & $1.66-2.08$ & 6 & 185 & 1.21 & $0.54-2.70$ \\
\hline Plumb & 309 & 11128 & 1.78 & $1.57-2.01$ & - & 37 & & 列 \\
\hline Welders and flame & 395 & 14288 & 1.91 & $1.71-2.14$ & 23 & 523 & 1.60 & $1.06-2.41$ \\
\hline Metal plating and coating work (656) & 22 & 598 & 2.71 & $1.78-4.12$ & 12 & 214 & 1.97 & $1.12-3.47$ \\
\hline Assemblers and other machine and metalware occupations (657) & 115 & 6113 & 1.29 & $1.07-1.56$ & 122 & 3445 & 1.25 & $1.04-1.49$ \\
\hline Electricians $(660)$ & 375 & 21285 & 1.24 & $1.10-1.39$ & 7 & 272 & 1.03 & $0.49-2.17$ \\
\hline Electronics and teleco & 118 & 7321 & 1.25 & $1.03-1.51$ & 15 & 706 & 0.90 & $0.54-1.49$ \\
\hline Telephone installation crew, linemen and cable joiners (664) & 116 & 5582 & 1.38 & 1.14-1.67 & 5 & 229 & 0.84 & $0.35-2.01$ \\
\hline quipment assemblers (665) & 21 & 1363 & 1.25 & $0.81-1.92$ & 188 & 5346 & 1.40 & $1.21-1.62$ \\
\hline Round-timber workers (670) & 19 & 654 & 1.70 & $1.08-2.67$ & 12 & 155 & 2.56 & $1.45-4.51$ \\
\hline Timberworkers (671) & 175 & 5505 & 2.05 & $1.75-2.40$ & 44 & 769 & 1.96 & $1.46-2.64$ \\
\hline Plywood and fiberboard workers (672) & 34 & 1314 & 1.75 & $1.24-2.46$ & 73 & 1412 & 1.77 & $1.41-2.23$ \\
\hline Construction $\mathrm{c}$ & 616 & 23966 & 1.59 & $1.44-1.75$ & 6 & 166 & 1.43 & $0.64-3.91$ \\
\hline Bench carpenters (675) & 42 & 1779 & 1.59 & $1.17-2.17$ & 5 & 124 & 1.53 & $0.64-3.67$ \\
\hline Cabinetmakers and joiners etc (676) & 98 & 4882 & 1.39 & $1.13-1.71$ & 10 & 550 & 0.67 & $0.36-1.25$ \\
\hline Woodworking machine operators, etc (677) & 86 & 3878 & 1.55 & $1.24-1.92$ & 30 & 692 & 1.49 & $1.04-2.13$ \\
\hline Painters, lacquerers and floor layers (680) & 324 & 10609 & 1.92 & $1.70-2.17$ & 34 & 843 & 1.45 & $1.03-2.03$ \\
\hline Bricklayers, plasterers and tile setters $(690)$ & 108 & 3648 & 1.87 & $1.54-2.27$ & 2 & 48 & 1.56 & $0.39-6.26$ \\
\hline Rod layers (692) & 29 & 1018 & 1.66 & $1.15-2.40$ & - & 4 & . & . \\
\hline Concrete shutterers & 43 & 1435 & 1.67 & $1.23-2.26$ & - & 10 & & . \\
\hline workers (694) & 11 & 338 & 2.11 & $1.16-3.82$ & - & 0 & . & . \\
\hline Insulation workers (695) & 60 & 2361 & 1.69 & $1.31-2.19$ & 3 & 74 & 1.37 & $0.44-4.26$ \\
\hline Assisting building workers and other assisting co & 431 & 16204 & 1.66 & $1.49-1.85$ & 34 & 713 & 1.62 & $1.16-2.27$ \\
\hline
\end{tabular}


Table 1. Continued.

\begin{tabular}{|c|c|c|c|c|c|c|c|c|}
\hline \multirow[t]{2}{*}{ Occupation a } & \multicolumn{4}{|c|}{ Men } & \multicolumn{4}{|c|}{ Women } \\
\hline & $\mathrm{N}$ & $\begin{array}{l}\text { Popula- } \\
\text { tion }^{5}\end{array}$ & $\mathrm{RR}^{\mathrm{c}}$ & $95 \% \mathrm{Cl}$ & N & $\begin{array}{l}\text { Popula- } \\
\text { tion }^{b}\end{array}$ & $\mathrm{RR}^{\mathrm{c}}$ & $95 \% \mathrm{Cl}$ \\
\hline \multicolumn{9}{|l|}{ Manufacturing and related work (continued) } \\
\hline Printers (701) & 46 & 3455 & 0.98 & $0.73-1.32$ & 27 & 584 & 1.72 & $1.18-2.51$ \\
\hline Grain millers $(720)$ & 18 & 451 & 2.40 & $1.51-3.83$ & 2 & 41 & 1.65 & $0.41-6.61$ \\
\hline Bakers (721) & 118 & 2236 & 3.78 & $3.13-4.57$ & 293 & 4080 & 2.54 & $2.26-2.86$ \\
\hline Chocolate and confectionery manufacturers (722) & 6 & 181 & 2.34 & $1.05-5.22$ & 22 & 556 & 1.38 & $0.91-2.10$ \\
\hline Brewers, beverage makers and kilnmen (723) & 14 & 445 & 2.14 & $1.27-3.63$ & 15 & 380 & 1.37 & $0.83-2.28$ \\
\hline Cannery workers (724) & 6 & 310 & 1.26 & $0.57-2.82$ & 19 & 339 & 2.03 & $1.29-3.18$ \\
\hline Butchers and sausage makers (725) & 74 & 2687 & 1.97 & $1.56-2.49$ & 23 & 1140 & 0.76 & $0.50-1.15$ \\
\hline Dairy workers $(726)$ & 24 & 713 & 2.30 & $1.54-3.44$ & 76 & 1802 & 1.41 & 1.13-1.77 \\
\hline Paper and cardboard mill workers (735) & 160 & 7930 & 1.35 & $1.15-1.59$ & 60 & 1411 & 1.46 & $1.13-1.88$ \\
\hline Refinery workers, other occupations in the chemical industry (736) & 81 & 3231 & 1.71 & $1.36-2.14$ & 43 & 1161 & 1.40 & $1.04-1.89$ \\
\hline Tobacco industry workers (740) & - & 28 & 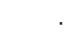 & & 11 & 171 & 2.20 & $1.22-3.98$ \\
\hline Plastic product workers (752) & 67 & 3134 & 1.56 & $1.22-2.00$ & 87 & 2059 & 1.52 & $1.23-1.88$ \\
\hline Tanners, fell mongers and pelt dressers (753) & 12 & 286 & 2.74 & $1.55-4.83$ & 18 & 318 & 1.88 & $1.19-2.99$ \\
\hline Musical instrument makers, etc (755) & 7 & 228 & 2.11 & $1.00-4.43$ & 2 & 63 & 1.26 & $0.31-5.03$ \\
\hline Concrete-mixer operators and cast concrete product workers (758) & 58 & 2430 & 1.58 & $1.21-2.05$ & 9 & 130 & 2.38 & $1.24-4.58$ \\
\hline Other occupations in manufacturing (759) & 35 & 1264 & 1.93 & $1.38-2.70$ & 55 & 1447 & 1.38 & $1.06-1.80$ \\
\hline Packers and labelers, etc (760) & 73 & 2691 & 1.74 & $1.38-2.21$ & 271 & 7427 & 1.24 & $1.09-1.40$ \\
\hline Crane operators, etc $(770)$ & 61 & 2349 & 1.56 & $1.21-2.02$ & 41 & 807 & 1.75 & $1.29-2.39$ \\
\hline Forklift operators, etc (771) & 148 & 5491 & 1.66 & $1.40-1.97$ & 20 & 528 & 1.33 & $0.86-2.07$ \\
\hline Construction machinery operators, etc (772) & 268 & 12946 & 1.32 & $1.16-1.51$ & 4 & 136 & 1.04 & $0.39-2.78$ \\
\hline Stationary engine and machinery operators (not on vessels) (773) & 118 & 4234 & 1.65 & $1.37-2.00$ & 12 & 262 & 1.66 & $0.94-2.92$ \\
\hline Maintenance crews and supervisors (774) & 45 & 1810 & 1.71 & $1.27-2.30$ & 19 & 425 & 1.61 & $1.03-2.53$ \\
\hline Machine setter operators (not in textile industry) and riggers (775) & 187 & 7222 & 1.69 & $1.45-1.97$ & 3 & 52 & 2.22 & $0.71-6.88$ \\
\hline Stevedores, etc (780) & 70 & 2933 & 1.53 & $1.20-1.95$ & 2 & 132 & 0.54 & $0.14-2.17$ \\
\hline Warehouse workers (781) & 310 & 13613 & 1.54 & $1.36-1.74$ & 199 & 4495 & 1.58 & $1.37-1.82$ \\
\hline Other manual workers $(790)$ & 127 & 4834 & 1.62 & $1.35-1.95$ & 42 & 821 & 1.80 & $1.33-2.43$ \\
\hline Service work (8) & 1177 & 51982 & 1.53 & $1.42-1.66$ & 6475 & 169193 & 1.41 & $1.35-1.46$ \\
\hline Firemen (800) & 81 & 4300 & 1.35 & $1.07-1.68$ & 1 & 37 & 1.32 & $0.19-9.36$ \\
\hline Policemen (801) & 148 & 7191 & 1.38 & $1.17-1.64$ & 13 & 325 & 1.91 & $1.11-3.29$ \\
\hline Customs officers and border guards (802) & 47 & 2663 & 1.19 & $0.89-1.60$ & 11 & 217 & 2.15 & $1.19-3.88$ \\
\hline Prison guards, etc (803) & 50 & 1562 & 2.03 & $1.53-2.69$ & 8 & 149 & 2.27 & $1.13-4.53$ \\
\hline Other guards (civil duties) (804) & 116 & 4572 & 1.65 & $1.36-1.99$ & 28 & 603 & 1.70 & $1.17-2.46$ \\
\hline Other occupations and guarding security (809) & 9 & 306 & 2.23 & $1.16-4.30$ & 11 & 193 & 2.21 & $1.22-3.99$ \\
\hline Cooks, etc (811) & 45 & 2553 & 1.63 & $1.21-2.20$ & 495 & 15390 & 1.23 & $1.12-1.35$ \\
\hline Kitchen assistants (812) & 9 & 384 & 2.01 & $1.04-3.87$ & 536 & 14778 & 1.33 & $1.22-1.46$ \\
\hline Housekeepers, child care in families and at home (813) & - & 29 & & ". & 718 & 22451 & 1.23 & 1.14-1.33 \\
\hline Home helps (municipal) (814) & 5 & 214 & 1.70 & $0.71-4.09$ & 451 & 12052 & 1.44 & $1.31-1.59$ \\
\hline Hotel and restaurant matrons $(816)$ & 1 & 65 & 1.55 & $0.22-11.0$ & 33 & 751 & 1.67 & $1.18-2.35$ \\
\hline Headwaiters, restaurant waiters(820) & 39 & 2695 & 1.26 & $0.92-1.74$ & 393 & 9815 & 1.62 & $1.46-1.80$ \\
\hline Waiters in bars and cafes, etc (821) & 14 & 743 & 1.40 & $0.83-2.37$ & 273 & 7404 & 1.36 & $1.20-1.53$ \\
\hline Building caretakers $(830)$ & 408 & 14787 & 1.65 & $1.48-1.84$ & 187 & 4720 & 1.37 & $1.19-1.59$ \\
\hline Cleaners and headcleaners (831) & 27 & 1776 & 1.13 & $0.77-1.66$ & 2414 & 53708 & 1.52 & $1.45-1.60$ \\
\hline Chimney sweeps (832) & 28 & 781 & 2.34 & $1.61-3.41$ & - & 3 & & . \\
\hline Hairdressers and barbers (840) & 10 & 372 & 2.09 & $1.12-3.89$ & 376 & 9494 & 1.61 & $1.45-1.79$ \\
\hline Beauticians and pedicurists (841) & - & 14 & & & 51 & 1513 & 1.39 & $1.05-1.83$ \\
\hline Laundry workers (850) & 16 & 360 & 2.96 & $1.81-4.84$ & 87 & 2505 & 1.19 & $0.96-1.47$ \\
\hline Pressing workers, etc, in laundries (851) & - & 9 & & & 11 & 161 & 2.07 & $1.14-3.73$ \\
\hline Hotel hall porters (890) & 41 & 1475 & 1.77 & $1.29-2.41$ & 20 & 992 & 0.94 & $0.60-1.45$ \\
\hline Burial service workers (892) & 8 & 306 & 1.64 & $0.82-3.28$ & 9 & 137 & 2.41 & $1.25-4.64$ \\
\hline Military work (9) & 112 & 9243 & 0.95 & $0.78-1.15$ & 2 & 121 & 0.82 & $0.21-3.28$ \\
\hline Unknown occupation (X) & 201 & 9781 & 1.36 & $1.17-1.58$ & 286 & 8535 & 1.27 & $1.13-1.43$ \\
\hline
\end{tabular}

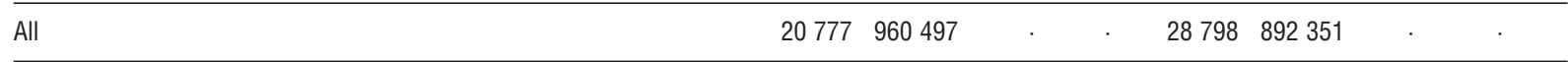

accupational code in parentheses.

${ }^{\mathrm{b}}$ Population = arithmetic mean of the employed population without preexisting asthma and between the ages of 25 and 59 years in the 1985, 1990, and 1995 censuses.

c Significantly increased relative risks are presented in boldface.

Among the men, the risk was significantly increased (5\% level) in 98 occupations (table 1). The risk was highest among bakers (RR 3.78), laundry workers (RR 2.96), shoemakers and repairers (RR 2.79), tanners, fell mongers and pelt dressers (RR 2.74), and metal plating and coating workers (RR 2.71). The study population covered the entire employed population. Therefore some of the occupation-specific risk estimates of table 1 
represent occupations in which the excess risk was not very high, but still statistically significant due to the large number of cases. Table 2 lists a more restricted selection of high-risk occupations (ie, those with the

Table 2. Restrictive selection of risk occupations for adult-onset asthma among employed Finnish men (25-59 years) in 19861998. Only occupations were included for which the lower limit of the $95 \%$ confidence interval $(95 \% \mathrm{Cl})$ of the relative risk (RR) exceeded 1.50 and there were at least 5 cases.

\begin{tabular}{|c|c|c|c|c|}
\hline Occupation a & N & $\begin{array}{l}\text { Popula- } \\
\text { tion }{ }^{b}\end{array}$ & $\mathrm{RR}$ & $95 \% \mathrm{Cl}$ \\
\hline Bakers (721) & 118 & 2236 & 3.78 & $3.13-4.57$ \\
\hline Laundry workers (850) & 16 & 360 & 2.96 & $1.81-4.84$ \\
\hline Shoemakers and shoe repairers (620) & ) 12 & 284 & 2.79 & $1.58-4.93$ \\
\hline $\begin{array}{l}\text { Tanners, fell mongers and } \\
\text { pelt dressers }(753)\end{array}$ & 12 & 286 & 2.74 & $1.55-4.83$ \\
\hline Metal plating and coating work (656) & 22 & 598 & 2.71 & $1.78-4.12$ \\
\hline Performing artists and vocalists (086) & ) 21 & 596 & 2.43 & $1.58-3.74$ \\
\hline Grain millers $(720)$ & 18 & 451 & 2.40 & $1.51-3.83$ \\
\hline Smiths (633) & 19 & 430 & 2.37 & $1.51-3.74$ \\
\hline Fur farmers (306) & 56 & 1435 & 2.35 & $1.80-3.08$ \\
\hline Chimney sweeps (832) & 28 & 781 & 2.34 & $1.61-3.41$ \\
\hline $\begin{array}{l}\text { Farmers, silviculturists, } \\
\text { horticulturists }(300)\end{array}$ & 2774 & 64180 & 2.32 & $2.17-2.48$ \\
\hline Agricultural workers (310) & 273 & 7323 & 2.31 & $2.03-2.64$ \\
\hline Dairy workers (726) & 24 & 713 & 2.30 & $1.54-3.44$ \\
\hline Miners, shot firers, etc (400) & 38 & 1049 & 2.16 & $1.56-2.98$ \\
\hline Timberworkers (671) & 175 & 5505 & 2.05 & $1.75-2.40$ \\
\hline Prison guards, etc (803) & 50 & 1562 & 2.03 & $1.53-2.69$ \\
\hline Butchers and sausage makers (725) & 74 & 2687 & 1.97 & $1.56-2.49$ \\
\hline $\begin{array}{l}\text { Painters, lacquerers and } \\
\text { floor layers (680) }\end{array}$ & 324 & 10609 & 1.92 & $1.70-2.17$ \\
\hline Welders and flame cutters (655) & 395 & 14288 & 1.91 & $1.71-2.14$ \\
\hline $\begin{array}{l}\text { Bricklayer } \\
\text { and tile se }\end{array}$ & 108 & 3648 & 1.87 & $1.54-2.27$ \\
\hline Sheet metal workers (653) & 385 & 14615 & 1.86 & $1.66-2.08$ \\
\hline Plumbers (654) & 309 & 11128 & 1.78 & $1.57-2.01$ \\
\hline
\end{tabular}

a Occupational code in parentheses.

b Population = arithmetic mean of the employed population without preexisting asthma and aged 25-59 years in the 1985, 1990, and 1995 censuses.

Table 3. Restrictive selection of risk occupations for adult-onset asthma among employed Finnish women (25-59 years of age) in 1986-1998. Only occupations were included for which the lower limit of the $95 \%$ confidence interval $(95 \% \mathrm{Cl})$ of the relative risk (RR) exceeded 1.50 and there were at least 5 cases.

\begin{tabular}{|c|c|c|c|c|}
\hline Occupation a & $\mathrm{N}$ & $\begin{array}{l}\text { Popula- } \\
\text { tion b }\end{array}$ & $\mathrm{RR}$ & $95 \% \mathrm{Cl}$ \\
\hline Shoemakers and shoe repairers $(620$ & 6 & 50 & 4.32 & $1.94-9.61$ \\
\hline $\begin{array}{l}\text { Railway and station personnel } \\
\text { (traffic department) (550) }\end{array}$ & 13 & 137 & 3.25 & $1.89-5.60$ \\
\hline Bakers (721) & 293 & 4080 & 2.54 & $2.26-2.86$ \\
\hline $\begin{array}{l}\text { Farmers, silviculturists, } \\
\text { horticulturists }(300)\end{array}$ & 1912 & 34070 & 1.90 & $1.80-2.00$ \\
\hline Agricultural workers (310) & 1227 & 20230 & 1.89 & $1.77-2.010$ \\
\hline
\end{tabular}

a Occupational code in parentheses.

b Population = arithmetic mean of the employed population without preexisting asthma and aged 25-59 years in the 1985, 1990 and 1995 censuses. lower limit of the $95 \%$ confidence interval of the RR exceeding 1.5 for the men). There were 22 such occupations. Of the 98 occupations with a significantly increased risk at the 5\% level among the men, 79 had a relative risk that was still significant at the $1 \%$ level.

Among the women, the risk was significantly increased in 72 occupations (table 1). The risk was highest among shoemakers and repairers (RR 4.32), railway and station personnel (RR 3.25), jewelry engravers (RR 2.77), engineroom crew (RR 2.58), molders (RR 2.56), round-timber workers (RR 2.56), and bakers (RR 2.54). Table 3 lists the occupations with the lower limit of the 95\% confidence interval of the relative risk exceeding 1.5 for the women. There were five such occupations. Of the 72 occupations with a significantly increased risk at the 5\% level among the women, 49 had a relative risk that was still significant at the $1 \%$ level.

In order to quantify the contribution of already established occupational risk factors in the overall workrelated asthma excess, we performed separate analyses excluding all cases recognized as occupational asthma. Recognized cases of occupational asthma contributed importantly to very few of the occupation-specific risk estimates. For male bakers, $42 \%$ of the total cases of asthma and, for some agricultural occupations, about $20 \%$ of the cases had been recognized as occupational asthma (tables 4 and 5). In occupations not presented in tables 4 and 5, the contribution of recognized cases of occupational asthma was negligible.

For the men there were seven occupations with a relative risk slightly, but statistically significantly below unity, and for the women there were eight such occupations. They included physicians, judges, automatic data processing occupations, teachers, mechanical engineers, and some other occupations, mainly in the major occupational group of technical, physical science, social science, humanistic, and artistic work. For both the men and the women, two of the occupation-specific relative risks were significantly below unity at the $1 \%$ level.

\section{Discussion}

The case identification was done through two national registers that rely on diagnoses that have been made by chest physicians on the basis of physiological criteria and that represent a persistent form of asthma. This definition of asthma (ie, clinical assessment) is the standard used to validate the other definitions used in epidemiologic studies (5). The examination of the asthma diagnoses (see the Methods section) indicated that the positive predictive value of our definition was very high, but, on the other hand, our definition only covered 
persistent forms of asthma. Consequently our results applied only to the work-related risk of persistent asthma.

We estimated the occupation-specific relative risks based on the occupation of the persons at the start of each of the 5-year follow-ups. Change of job due to respiratory symptoms is frequent before the diagnosis of asthma (6). Such changes would cause bias towards unity because people would probably move from occupations with exposure to occupations with less exposure. The assumption of a lack of exposure in our reference group is not fully tenable (eg, exposure to paper dust, emissions from office equipment or other impurities due to poor ventilation), however, and would again have caused underestimation of the occupation-specific risk estimates in the remaining occupations. Exposure to workplace air pollution occurs more often in occupations associated with a low socioeconomic status. Such an association could introduce confounding due to lifestyle factors or housing environment and possibly result in overestimation of the work-related risk. A separate analysis using lower-level administrative workers as a reference group did not indicate such confounding of the work-related risk estimates.

There is conflicting evidence as to whether smoking is a risk factor for adult-onset asthma, although there are more studies showing no association (7-11). Recent studies of the work-related risks of asthma have reported that adjustment for smoking had only a minimal or no effect on the risk estimates $(6,11-13)$. We could not adjust for smoking, but it is unlikely that differences in the prevalence of smoking between occupations would have significantly biased our risk estimates.

Only a few population-based studies have previously estimated occupation-specific risks of asthma. The

Table 4. Comparison of the occupation-specific relative risk of asthma with and without recognized cases of occupational asthma among Finnish men in 1986-1998. Only occupations were included for which occupational asthma accounted for at least $10 \%$ of the cases and there were at least 5 cases. $\left(95 \% \mathrm{Cl}=95 \%\right.$ confidence interval, $\mathrm{RR}_{1}=$ relative risk based on all cases of asthma, $R R_{2}=$ relative risk after the exclusion of cases of occupational asthma)

\begin{tabular}{|c|c|c|c|c|c|c|c|}
\hline \multirow[t]{2}{*}{ Occupation a } & \multirow[t]{2}{*}{$\begin{array}{l}\text { Total asthma } \\
\text { cases (N) }\end{array}$} & \multicolumn{2}{|c|}{$\begin{array}{l}\text { Recognized cases } \\
\text { of occupational } \\
\text { asthma }\end{array}$} & \multirow[t]{2}{*}{$\mathrm{RR}_{1}$} & \multirow[t]{2}{*}{$95 \% \mathrm{Cl} b$} & \multirow[t]{2}{*}{$\mathrm{RR}_{2}$} & \multirow[t]{2}{*}{$95 \% \mathrm{Cl}^{\mathrm{c}}$} \\
\hline & & N & $\%$ & & & & \\
\hline Farmers, silviculturists, horticulturists (300) & 2774 & 664 & 24 & 2.32 & $2.27-2.48$ & 1.76 & $1.64-1.89$ \\
\hline Fur farmers (306) & 56 & 12 & 21 & 2.35 & $1.80-3.08$ & 1.87 & $1.39-2.53$ \\
\hline Agricultural workers (310) & 273 & 52 & 19 & 2.31 & $2.03-2.64$ & 1.90 & $1.65-2.19$ \\
\hline Painters, lacquerers and floor layers (680) & 324 & 30 & 9 & 1.92 & $1.70-2.17$ & 1.77 & $1.56-2.01$ \\
\hline Bakers (721) & 118 & 50 & 42 & 3.78 & $3.13-4.57$ & 2.13 & $1.74-2.83$ \\
\hline Butchers and sausage makers (725) & 74 & 14 & 19 & 1.97 & $1.56-2.49$ & 1.63 & $1.26-2.12$ \\
\hline Plastic product workers (752) & 67 & 10 & 15 & 1.56 & $1.22-2.00$ & 1.36 & $1.04-1.77$ \\
\hline Cooks, etc (811) & 45 & 5 & 11 & 1.63 & $1.21-2.20$ & 1.51 & $1.10-2.07$ \\
\hline
\end{tabular}

a Occupational code in parentheses.

b $95 \% \mathrm{Cl}$ for $\mathrm{RR}_{\text {. }}$.

c $95 \% \mathrm{Cl}$ for $\mathrm{RR}_{2}$.

Table 5. Comparison of the occupation-specific relative risk of asthma with and without recognized cases of occupational asthma among Finnish women in 1986-1998. Only occupations were included for which occupational asthma accounted for at least $10 \%$ of the cases and there were at least 5 cases. $\left(95 \% \mathrm{Cl}=95 \%\right.$ confidence interval, $\mathrm{RR}_{1}=$ relative risk based on all cases of asthma, $\mathrm{RR}_{2}=$ relative risk after the exclusion of cases of occupational asthma)

\begin{tabular}{|c|c|c|c|c|c|c|c|}
\hline \multirow[t]{2}{*}{ Occupation a } & \multirow[t]{2}{*}{$\begin{array}{l}\text { Total asthma } \\
\text { cases }(N)\end{array}$} & \multicolumn{2}{|c|}{$\begin{array}{l}\text { Recognized cases } \\
\text { of occupational } \\
\text { asthma }\end{array}$} & \multirow[t]{2}{*}{$\mathrm{RR}_{1}$} & \multirow[t]{2}{*}{$95 \% \mathrm{Cl}^{\mathrm{b}}$} & \multirow[t]{2}{*}{$\mathrm{RR}_{2}$} & \multirow[t]{2}{*}{$95 \% \mathrm{Cl} c$} \\
\hline & & $\mathrm{N}$ & $\%$ & & & & \\
\hline Farmers, silviculturists, horticulturists (300) & 1912 & 463 & 24 & 1.90 & $1.80-2.00$ & 1.42 & $1.34-1.51$ \\
\hline Livestock breeders (305) & 23 & 5 & 22 & 1.94 & $1.29-2.93$ & 1.52 & $0.96-2.42$ \\
\hline Agricultural workers (310) & 1227 & 276 & 23 & 1.89 & $1.77-2.01$ & 1.45 & $1.35-1.55$ \\
\hline Plywood and fiberboard workers (672) & 73 & 9 & 12 & 1.77 & $1.41-2.23$ & 1.55 & $1.21-1.98$ \\
\hline Painters, lacquerers and floor layers (680) & 34 & 5 & 15 & 1.45 & $1.03-2.03$ & 1.23 & $0.86-1.78$ \\
\hline Bakers (721) & 293 & 77 & 26 & 2.54 & $2.26-2.86$ & 1.86 & $1.62-2.13$ \\
\hline Plastic product workers (752) & 87 & 10 & 12 & 1.52 & $1.23-1.88$ & 1.35 & $1.07-1.69$ \\
\hline
\end{tabular}

a Occupational code in parentheses.

b $95 \% \mathrm{Cl}$ for $\mathrm{RR}_{1}$.

c $95 \% \mathrm{Cl}$ for $\mathrm{RR}_{2}$. 
European Community Respiratory Health Survey calculated odds ratios for 30 aggregated sets of occupations based on a population sample of 15637 people aged 2044 years (14). A case-referent study from Singapore (787 cases, 1591 referents, aged 20-54 years) reported odds ratios for 9 major occupational groups and 30 of their subcategories according to the national classification of occupations (15). Due to the size of the studies, both used a classification of occupations that was less detailed than the one used by us. In addition, even at a broader level, the classifications were not fully comparable with each other or with ours. Nevertheless some comparisons can be made. Farmers and agricultural workers, as well as cleaners, had a roughly twofold relative risk in both of the other studies as well as in ours. In addition, the risks observed for manufacturing occupations in the metal industry, for the wood industry, for the food industry, for plastics production, and for welders and painters were similar between the European Community Respiratory Health Survey and our study.

Our epidemiologic approach to work-related asthma excess is broader than the definition of occupational asthma used for most surveillance or medicolegal purposes (16). As in a previous Finnish study (17), recognized cases of occupational asthma accounted for only $5 \%$ of all cases of adult-onset asthma in our study, and their contribution to the overall excess of asthma was minor for occupations other than bakers and some agricultural occupations. Most of the cases of occupational asthma in Finland (including those in our study) are related to exposure to sensitizing agents like flour dust and animal epithelia (18). In general it can be concluded that, with respect to occupational asthma, well-established causative agents are more likely to be compensated and reported and it is easier to justify causality at the individual level for sensitizing than for nonsensitizing agents. Therefore the information on workrelated asthma excess from compensation or notification systems for occupational asthma is likely to be biased towards well-established sensitizing causative agents. For many of the risk occupations of our study, exposure to irritative indoor or outdoor pollutants or factors (eg, dusts, welding or soldering fumes, disinfectants, components of traffic or combustion exhaust, cold air, etc) may be more a plausible explanation for the excess risk. The aim of our study was not, however, to study specific work-related causative factors of asthma, but instead to determine systematically the occupationspecific relative risks. It is interesting, however, that, even for well-established risk occupations of sensitizer-related occupational asthma, the cases recognized for occupational asthma had only a minor contribution to the overall excess in asthma incidence.

Our only a priori assumption with respect to the risk occupations was that persons in administrative and clerical work were considered "unexposed" to harmful air pollution at work. In other words, all the remaining occupations were considered as potentially having some harmful exposure, and a large number of comparisons was thereafter made to estimate the relative risk. In explorative studies covering a large number of occupations, some of the "significant" results are likely to be due to chance rather than due to true associations. In independent testing at the significance level of 5\%, only $7(2.5 \%)$ of the 275 occupation-specific tests of nonadministrative workers would become "significant" in both directions merely by chance (or one or two occupations at the $1 \%$ level). For the reasons already pointed out, the relative risks observed in our study were, however, more likely to be underestimates than overestimates of the true relative risks. For most of the identified risk occupations, the risk was increased for both genders. It is also noteworthy that the major occupational groups of technical, physical science, social science, humanistic, and artistic work and sales work were not defined a priori as unexposed, because, although most of the workers in these occupations work under conditions resembling those of administrative and clerical work, in some of the occupations, specific exposures may occur (eg, laboratory work, pharmacies). It is noteworthy, however, that finally the overall relative risk of these major occupational groups and most of their subcategories was close to that of the reference group. Interestingly, the numbers of "significant" low-risk occupations found for each gender corresponded to the preceding estimates of the "chance" findings. Yet there may as well have been other reasons for the observed low risk, for example, a reduced need to seek reimbursement rights (physicians) or out-selection of symptomatic persons during obligatory military service (military work).

Our study is the first population-based nationwide study that has systematically determined the occupationspecific incidence and relative risk of adult onset asthma. The study included the entire employed workforce of Finland and covered 13 years and nearly 50000 incident cases of asthma. Consequently it was possible to estimate the risks at a much more detailed level of occupational classification than before. A large number of risk occupations was identified, especially in manufacturing, service, and agricultural work. This information will be used to target studies focusing on the determinants of the asthma excess and to explore possibilities for better asthma control among asthmatics working in risk occupations.

\section{Acknowledgments}

The study was financially supported by the Rehabilitation Funds of the Finnish Social Insurance Institution. 
We are indebted to Marianne Johnson of Statistics Finland, Kari Toivola and Timo Pitkonen of the Finnish Social Insurance Institution and Anja Savela of the Finnish Register of Occupational Diseases for their assistance in building the data files.

\section{References}

1. Venables KM, Chan-Yeung M. Occupational asthma. Lancet 1997;349:1465-69.

2. Pearce N, Beasley R, Burgess C, Crane J. Asthma epidemiology: principles and methods. New York (NY): Oxford University Press, 1998.

3. Karjalainen A, Kurppa K, Martikainen A, Klaukka T, Karjalainen J. Work is related to a substantial portion of adultonset asthma incidence in the Finnish population. Am J Respir Crit Care Med 2001;164:565-8.

4. de Marco R, Locatelli F, Sunyer J, Burney P. Differences in incidence of reported asthma related to age in men and women: a retrospective analysis of the data of the European respiratory health survey. Am J Respir Crit Care Med 2000; 162:68-74.

5. Pekkanen J, Pearce N. Defining asthma in epidemiological studies. Eur Respir J 1999;14:951-7.

6. Kogevinas M, Anto JM, Soriano JB, Aurelio T, Burney P. The risk of asthma attributable to occupational exposures: a population-based study in Spain. Am J Respir Crit Care Med 1996;154:137-43.

7. Vesterinen E, Kaprio J, Koskenvuo M. Prospective study of asthma in relation to smoking habits among 14729 adults. Thorax 1988;43:534-9.

8. Troisi RJ, Speizer FE, Rosner B, Trichopoulos D, Willett
WC. Cigarette smoking and incidence of chronic bronchitis and asthma in women. Chest 1995;108:1557-61.

9. Flodin E, Jönsson P, Ziegler J, Axelson O. An epidemiologic study of bronchial asthma and smoking. Epidemiology 1995;6:503-5.

10. Siroux V, Pin I, Oryszczyn MP, Le Moual N, Kauffman F. Relationship of active smoking to asthma and asthma severity in the EGEA study. Eur Respir J 2000;15:470-7.

11. Melbostad E, Eduard W, Magnus P. Determinants of asthma in a farming population. Scand J Work Environ Health 1998;24:262-9.

12. Toren K, Balder B, Brisman J, Lindholm N, Löwhagen O, Palmqvist $\mathrm{M}$, et al. The risk of asthma in relation to occupational exposures: a case-control study from a Swedish city. Eur Respir J 1999;13:496-501.

13. Flodin U, Ziegler J, Jönsson P, Axelson O. Bronchial asthma and air pollution at workplaces. Scand J Work Environ Health 1996;22:451-6.

14. Kogevinas M, Anto JM, Soriano JB, Tobias A, Burney P. Occupational asthma in Europe and other industrialised areas: a population-based study. Lancet 1999;353:1750-4.

15. Ng TP, Hong CY, Goh LG, Wong ML, Koh KTC, Ling SL. Risk of asthma associated with occupations in a communitybased case-control study. Am J Ind Med 1994;25:709-18.

16. Wagner GR, Wegman DH. Occupational asthma: prevention by definition. Am J Ind Med 1998;33:427-9.

17. Reijula K, Haahtela T, Klaukka T, Rantanen J. Incidence of occupational asthma and persistent asthma in young adults has increased in Finland. Chest 1996;110:58-61.

18. Karjalainen A, Kurppa K, Virtanen S, Keskinen H, Nordman $\mathrm{H}$. Incidence of occupational asthma by occupation and industry in Finland. Am J Ind Med 2000;37:451-8.

Received for publication: 11 September 2002 